\title{
Time Shared Optical Network (TSON): A Novel Metro Architecture for Flexible Multi-Granular Services
}

\author{
G. S. Zervas ${ }^{1}$, J. Triay ${ }^{12}$, N. Amaya ${ }^{1}$, Y. Qin ${ }^{1}$, C. Cervelló - Pastor $^{2}$, D. Simeonidou ${ }^{1}$ \\ ${ }^{1}$ School of Computer Science and Electronic Engineering, University of Essex, Colchester CO4 3SQ, U.K \\ ${ }^{2}$ Department of Telematics Engineering, Universitat Politècnica de Catalunya (UPC), Castelldefels, 08860, Spain \\ gzerva@essex.ac.uk
}

\begin{abstract}
This paper presents the TSON metro mesh network architecture for guaranteed, statistically-multiplexed services. It proposes tunable time-wavelength assignment, one-way treebased reservation and node architecture. Results demonstrate high network efficiency, fast service delivery and guaranteed QoS.

OCIS codes: (060.4250) Networks; (060.4510) Fiber optics communications
\end{abstract}

\section{Introduction}

A broad range of emerging services and applications (wide-range of multi-media, distributed applications such as Cloud, etc.) are driving the growing trend of network traffic with increasing demand for high bandwidth and flexibility. In addition, such applications require guaranteed multi-granular short-lived services i.e., from seconds to minutes with bandwidths from sub-wavelength to multi-wavelength. In order to provide these services, a new architecture is required that can support multiple end-to-end lightpaths (LPs) over single (or multiple) wavelength(s) and deliver dynamic access to transparent multi-granular flows as a guaranteed (no contention) network service.

Optical packet switching (OPS) and optical burst switching (OBS) have been proposed to support subwavelength services [1]. However, these techniques do not provide guaranteed bandwidth services. Recently, TimeDriven-Switched Optical Network [2] has proposed to deliver sub-wavelength switched synchronous virtual pipes. To guarantee such services, this approach uses a synchronous global common time reference from Galileo or GPS. Also, LOBS-H [3] is an alternative solution that allocates wavelength sharable home circuits for each sourcedestination pair. These circuits provide guaranteed bandwidth for conforming traffic that originates from the same source to different destinations and also allow for non-guaranteed statistically multiplexed non-conforming traffic from any source to any destination. Furthermore, there has been considerable effort on routing, wavelength and time assignment (RWTA) algorithms to calculate two-way reserved Time Division Multiplexed (TDM) wavelength services [4]. It is also worth noting that current approaches consider ring solutions [1,5] for metro. This introduces additional complexity on how to interconnect all nodes (e.g. interconnected rings) and deliver the bandwidth services over topologies such as the one portrayed in Fig. 1.a that have an inherit mesh multi-degree connectivity.

In this paper, a novel optical network solution is proposed - the Time Shared Optical Network (TSON) - to deliver both highly flexible statistically multiplexed optical network infrastructure and on-demand guaranteed contention-free time-shared multi-granular services. It supports traffic flows from any source to any destination in transparent optical networks for the metro region supporting the physical interconnection requirements. The architecture is based on user/application-driven bandwidth service requests, centralized RWTA calculation, and oneway tree-based provisioning that allows for flexible symmetric/asymmetric multi-granular bandwidth services with the use of either fixed or tunable transceivers. It delivers contention-free optical switching and transport of contiguous and non-contiguous time-slices across one or multiple wavelengths per service. It also doesn't require global synchronization, optical buffering and wavelength conversion, thus, reducing implementation complexity.

\section{Time-Shared Optical Network (TSON) network, node architecture and protocol design for metro region}

The Time Shared Optical Network (TSON) as shown in Fig. 1b handles three time-scales for resource reservation: a) Time-Slice, b) Frame, c) Connection. Time-slices have a duration of $10 \mu \mathrm{s}$ and are the minimum resource unit per wavelength that can be transparently switched across the network. Multiple time-slices form a 1-ms frame. The actual number of time-slices in a frame depends on the inter-slice gap. For instance, if a worst-case scenario of 1- $\mu$ s inter time-slice gap is considered to account for switching-time and clock recovery, the network can still achieve $90 \%$ efficiency. Finally, the connection represents the duration of the lightpath requested by the user. The minimum bandwidth and granularity is $100 \mathrm{Mbps}$ and the maximum depends on the total capacity of the network (e.g. 160Gbps on a 16 wavelength @ 10Gbps). As shown in Fig. 1b, a single LP might reserve sufficient number of contiguous/non-contiguous time-slices from one or more wavelengths to meet the bandwidth service demand.

Fig. 1c shows the TSON architecture. The architecture consists of a central server for RWTA and one-way treebased provisioning initialization. As such, this is the only service blocking point, which simplifies provisioning. 


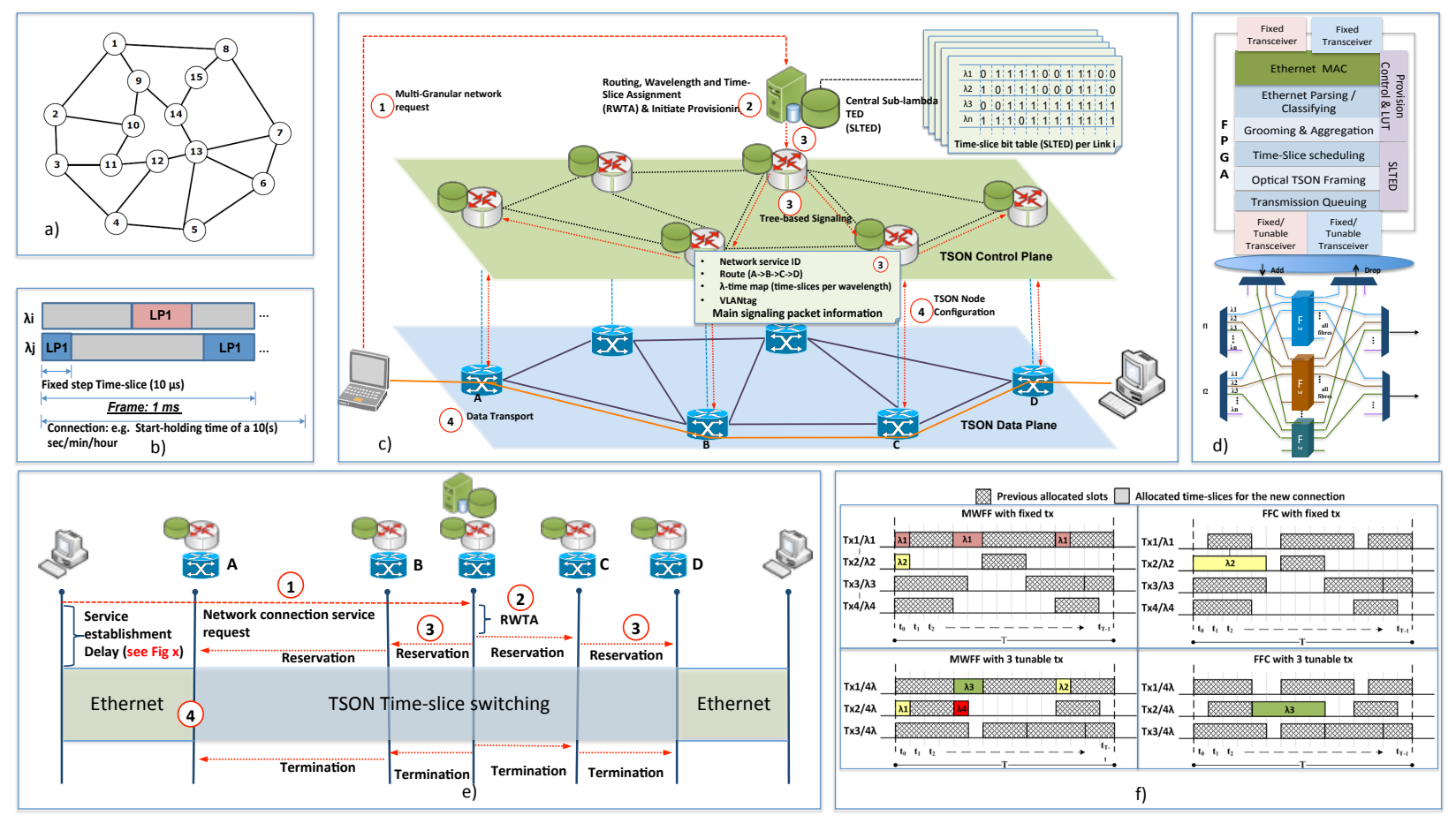

Fig. 1 a) Telefonica's Madrid Metro topology, b) Resource time-scales granularity, c) TSON metro network architecture, d) TSON Node architecture, e) end-to-end service provisioning flow, and f) proposed RWTA policies

However, it is also a single point of failure and as such a redundant server on a different node is needed. The TSON node architecture (Fig. 1d) that is under development to construct a complete TSON testbed consists of two main functional entities.The FPGA-based TSON line card (based on Xilinx Virtex6-HXT) performs the Layer 2 functions for ingress traffic and the fast $\lambda$-modular OXC (based on bit-rate independent 10-ns PLZT $4 \times 4$ switch [6] and shown in Fig. 1d) switches time-slices transparently along the end-to-end multi-granular lightpath. The line card performs Ethernet frame parsing on incoming traffic. According to the allocated time-slices per wavelength for a particular connection provided through the provisioned control packet the Ethernet frames are classified and aggregated in buffers to fill time-slices. The formed time-slice data sets are encapsulated/framed and fed to transmission queues (one per transceiver) to be transported on the allocated wavelength/time-slices within a frame. Transceivers can either be fixed or tunable. When deploying tunable transceivers, by use of 70 -ns tunable lasers and fixed receivers, different time-slices from the same transceiver can be carried over different wavelengths.

The service provisioning mechanism across the network is shown in Fig. 1e. A client sends a network service request to central TSON server (1). Then, the server performs RWTA by accessing the sub-lambda trafficengineering database (SLTED) (2). The SLTED maintains updated link state information for the whole metro network and holds a $\lambda$-time bitmap resource representation (time-slices per lambda per frame per port). One-bit represents a unique time-slice per wavelength. Such information is used by the RWTA to first compute the bandwidth availability of the candidate routes and then assign the finer contiguous or non-contiguous time-slices across one or multiple wavelengths on the selected path. Such calculation considers the wavelength and the timeslice continuity constraint due to lack of wavelength conversion and time-slice interchange. After the calculation of RWTA, a provisioning control packet is constructed. It carries the following information: a) Network service ID, b) Route (A->B->C->D), c) time bitmap table, and d) VLANtag. The VLANtag is required to associate the provisioned multi-granular lightpath to the expected incoming flow. The control packet is signaled to all required nodes on a tree-based fashion (3). Since this originates from a central point of the metro network the service provisioning time is considerably reduced compared to standard two-way reservation systems (e.g. GMPLS). After a maximum provisioning time (based on processing and propagation times) the client can start transmitting data (4).

\section{Simulation and results}

The proposed architecture has been evaluated through simulations using Telefonica's Madrid metro reference network as shown in Fig. 1a. The network comprises 15 nodes and 23 bidirectional links with 16 wavelengths per link and $10 \mathrm{~Gb} / \mathrm{s}$ per wavelength. Each node operates as both core (with an OXC) and edge (with add\&drop ports). In such a scenario, there are 16 wavelength per link and $10 \mathrm{Gbps}$ per channel. Wavelength conversion, optical buffering and time-slice interchange are not deployed in the network. Also, a fixed time-slice approach has been 
followed with a time-slice and frame size of $10 \mu \mathrm{s}$ and $1 \mathrm{~ms}$, respectively. With respect to the traffic characteristics, connection arrivals follow a Poisson process and exponential holding time with mean $1 / \mu=60 \mathrm{~s}$. A service connection of $1 \mathrm{Gbps}$ has been employed. Finally, the RWTA is implementing two different time-slice assignment policies namely, first, first-fit contiguous (FFC) and multi-wavelength first-fit (MWFF). FFC constraints the timeslice allocation per connection to be contiguous (no fragmentation) and on a single wavelength; whereas MWFF is more flexible by allowing non-contiguous time-slice assignment on multiple wavelengths using one or multiple transceivers (Fig. 1f). Also, we have tested two ingress port configurations: one with 16 fixed transceivers, and another with 12 tunable transceivers. The four proposed allocation scenarios are shown in Fig. 1f.

Fig. 2a shows the connection blocking probability as a function of the offered load to the network. FFC allocation delivers similar results when ingress TSON nodes have either 12 tunable transceivers or 16 fixed transceivers i.e. a $25 \%$ port-count reduction when tunable transceivers are used. Furthermore, by using the MWFF, same blocking probability (10E-3) can be achieved for an increased offered load (60\% more) due to the greater time-slice allocation flexibility. Also, it is evident that MWFF can benefit more from higher number of fixed ports than transceiver tunability as shown in Fig. 2a, since the algorithm itself allocates time-slices per connection in both time and frequency domain (algorithm tunability). Additional results (Fig. 2b) show that, as expected for the FFC case, the number of fragments per connection remains one, regardless of the offered load or transceiver type. In contrast, MWFF uses more than 8 fragments for all the levels of offered load considered. Moreover, as shown in Fig. 2c, MWFF uses more wavelengths (up to 6) as the offered load increases to provide the required number of time-slices. Also, under maximum offered load the add port utilization for FFC reaches $68 \%$ with fixed and $63 \%$ with tunable transceivers, whereas for MWFF it reaches $82 \%$ with fixed and $72 \%$ with tunable transceivers (Fig.2d). Hence, MWFF provides up to $32 \%$ higher add-port utilization compared to FFC. Thus, MWFF guarantees lower blocking and increased throughput at the expense of heavily fragmenting the time-slice allocation across multiple wavelengths. Therefore, there is a tradeoff between blocking probability and implementation challenges that relates to electronic aggregation/grooming and optical time-slice switching. Finally, results (not shown) demonstrate that the maximum average No. of hops is 2.6 and the maximum e-2-e Ethernet frame delay is $2 \mathrm{~ms}$ for all approaches.
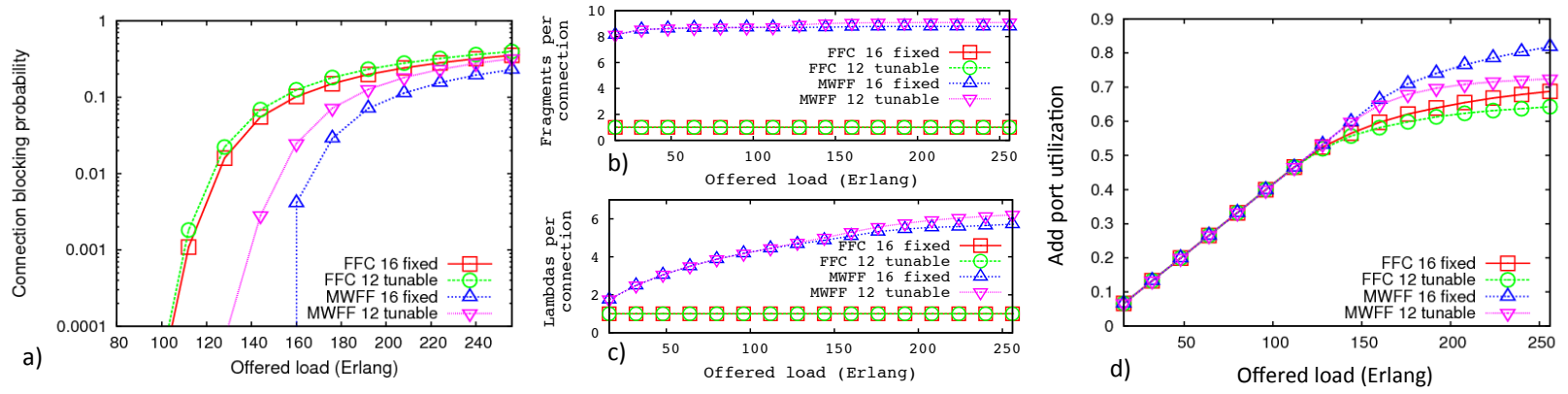

Fig. 2 Results of TSON on a metro network with 16 wavelengths as function of offered load: a) connection blocking probability, b) average number of non-contiguous time-slice fragments per connection and c) average number of lambdas per connection, and d) add port utilization.

\section{Conclusions}

This paper reports on a novel architecture, Time-Shared Optical Network (TSON) that enables guaranteed contention-free optical multi-granular services on metro mesh networks. Two routing, wavelength and time-slice assignment policies using both fixed and tunable ingress transceivers are proposed and evaluated. The MWFF algorithm delivers $60 \%$ increased network efficiency and 32\% improved add-port utilization compared to FFC when fixed transceivers are considered. To deliver such performance a number of fragmented time-slices and multiple wavelengths have to be allocated for each connection. However, the deployment of tunable transceivers on TSON nodes can reduce the total number of transceivers by $25 \%$ in case of FFC time-slice policy for almost the same performance and reduce the implementation complexity due to non-fragmented allocation of time-slices on a single wavelength. Finally, the proposed overall architecture delivers $2 \mathrm{~ms}$ Ethernet-frame end-to-end delay.

\section{Acknowledgements}

This work has been supported by the EC through IST STREP project MAINS (INFSO-ICT-247706).

\section{References}

[1] D. Chiaroni, et.al., "Demonstration of the Interconnection of Two Optical Packet Rings with a Hybrid ...", PD3.5, ECOC 2010

[2] F. Vismara, et.al. , "A Comparative Blocking Analysis for Time-Driven-Switched Optical Networks", ONDM 2011

[3] M. A. Gonzalez-Ortega, et.al, "LOBS-H: An Enhanced OBS with Wavelength Sharable Home Circuits", ICC 2010

[4] B. Wen, et.al, "Routing, wavelength and time-slot-assignment algorithms for wavelength-routed optical WDM/TDM networks", ICTON 2010

[5] Dunne, J., "Optical Packet Switch and Transport: A New Metro Platform to Reduce Costs and Power by 50\% to 75\% ...", WOBS 2009

[6] K. Nashimoto, et.al., "Nano-Second Response, Polarization Insensitive and Low-Power Consumption PLZT 4x4 ...”, OTh3, OFC 2011 